Cinémas

Revue d'études cinématographiques

Journal of Film Studies

\title{
Jouer aux Indiens : In the Land of the Headhunters ou War Canoes
}

\section{Catherine Russell}

Volume 6, numéro 1, automne 1995

Le cinéma muet au Québec et au Canada : nouveaux regards sur une pratique culturelle

URI : https://id.erudit.org/iderudit/1000958ar

DOI : https://doi.org/10.7202/1000958ar

Aller au sommaire du numéro

Éditeur(s)

Cinémas

ISSN

1181-6945 (imprimé)

1705-6500 (numérique)

Découvrir la revue

Citer cet article

Russell, C. (1995). Jouer aux Indiens : In the Land of the Headhunters ou War Canoes. Cinémas, 6(1), 33-46. https://doi.org/10.7202/1000958ar
Résumé de l'article

Le film d'Edward Curtis réalisé en 1915 et relatif aux Indiens kwakiutls de l'île de Vancouver est analysé dans la perspective du cinéma des premiers temps (" primitif ") et de l'avant-garde. Le film original, In the Land of the Headhunters, est recontextualisé et comparé à Nanook of the North. Le film restauré, In the Land of the War Canoes, est considéré dans la perspective de l'ethnographie du spectatorat et de la réappropriation, par les aborigènes, des textes colonialistes. En tant qu'allégorie du " paradigme sauvage ", l'ethnographie de Curtis est considérée à l'intérieur d'un discours postmoderniste sur la représentation culturelle. 


\title{
Jouer aux Indiens: In the Land of the Headhunters ou War Canoes ${ }^{1}$
}

\section{Catherine Russell}

\begin{abstract}
RÉSUMÉ
Le film d'Edward Curtis réalisé en 1915 et relatif aux Indiens kwakiutls de l'île de Vancouver est analysé dans la perspective du cinéma des premiers temps ("primitif") et de l'avant-garde. Le film original, In the Land of the Headhunters, est recontextualisé et comparé à Nanook of the North. Le film restauré, In the Land of the War Canoes, est considéré dans la perspective de l'ethnographie du spectatorat et de la réappropriation, par les aborigènes, des textes colonialistes. En tant qu'allégorie du "paradigme sauvage", l'ethnographie de Curtis est considérée à l'intérieur d'un discours postmoderniste sur la représentation culturelle.
\end{abstract}

\section{ABSTRACT}

Edward Curtis's 1914 films of the Kwakiutl Indians of Vancouver Island is analyzed from the perspective of early cinema and the avant-garde. The original film, In the Land of the Headhunters, is recontextualized and compared both to Nanook of the North; the restored film, In the Land of the War Canoes, is considered from the perspective of ethnographic spectatorship and native reappropriation of colonialist texts. As an allegory of the "salvage paradigm," Curtis's ethnography is positioned within a postmodernist discourse on cultural representation.

La théorie contemporaine remet en question nombre des conventions de la représentation ethnographique. Les suppositions épistémologiques et esthétiques de l'anthropologie 
moderne étaient solidement basées sur le discours colonialiste considéré comme un art et une science pendant la première moitié du XX⿳亠丷厂 siècle. Même si personne n'a su décrire une recette qui définisse une forme de représentation ethnographique "correcte ", c'est tout de même l'élan vers le "correct " qui a plongé si profondément le cinéma ethnographique dans l'esthétisme du réalisme. Aujourd'hui, l'analyse des complexités du discours ethnographique a ouvert la voie à l'ethnographie expérimentale comme forme valable de praxis culturelle. Le développement de cette ethnographie expérimentale dépend en partie de " [...] l'utilisation des textes existants comme étalon de mesure" (Manganaro, p. 12), ou sites du jeu et du pouvoir discursifs.

Dans l'histoire du cinéma, il existe un riche terrain d'ethnographie expérimentale qui n'attend qu'à être reconnu comme tel. James Clifford soutient que les structures allégoriques de la lecture et de l'écriture représentent le moyen de mettre en évidence les paradigmes de l'authenticité, du salvage et du pastoral, dans la représentation ethnographique (Clifford, 1986, p. 119) ${ }^{2}$. C'est dans l'esprit de cette représentation allégorique que j'entends analyser le film In The Land of the War Canoes. Seule la critique post-structuraliste de l'anthropologie peut permettre, dans un contexte postmoderne, de revisiter ce film de 1914 en tant que forme particulière de pastiche, riche en enseignements pour la vidéo et le cinéma contemporains. Non seulement le film s'éloigne-t-il de toutes normes d'ethnographie, il défie aussi toute distinction entre le cinéma des premiers temps et la narrativité postgriffithienne; il peut être associé aux actualités Lumière tout autant qu'aux modes fictionnels de Méliès.

En 1914, Edward Curtis, en étroite collaboration avec les Indiens kwakiutls de l'île de Vancouver, tentait de créer un "scénario" ou un drame narratif qui serait à la fois divertissant et instructif. Ce fut un échec cuisant sur les deux fronts et le film tomba vite dans l'oubli. En 1973, deux anthropologues, Bill Holm et George Quimby, avec la participation des Kwakiutls, ont restauré le film en ajoutant une trame sonore et en changeant le titre original de Curtis, In the Land of the Headhunters, pour In the Land of the War Canoes. Mon analyse porte donc sur deux films différents. Headhunters est, en soi, un film perdu. Il 
n'a tenu l'affiche que très brièvement ${ }^{3}$, et une fois entreposé, il a été très endommagé par la négligence et par le feu, si bien que des scènes entières et un grand nombre de plans ont été détruits. Mes réflexions sur ce film perdu sont donc hypothétiques; elles s'appuient sur les segments repérables dans le film "restauré". Elles sont par ailleurs formulées dans la perspective d'une forme rédemptrice d'ethnographie, inspirée par la réappropriation virtuelle du film par les Kwakiutls.

L'échec commercial de Headhunters a pu dépendre, en partie, du fait que Curtis, jouissant alors d'une grande réputation de photographe, était apparemment dépassé par l'évolution des conventions du récit cinématographique. Ses séquences évoquaient bien plus les films de Méliès, d'Edison et de Porter que ceux réalisés en 1914. Ironiquement, Curtis s'était inspiré du succès énorme des films d'Indiens tournés par D. W. Griffith à la Biograph. De 1908 à 1914, Griffith a réalisé au moins 18 films mettant en vedette des acteurs blancs dans le rôle d'Indiens. Certains de ses films dépeignent les Indiens comme des sauvages dévastateurs; toutefois, plusieurs les présentent comme des gens sympathiques, victimes des Blancs. Certains mélodrames romantiques sont même entièrement mis en scène dans des collectivités indiennes, sans aucun personnage "blanc ${ }^{4}$ ".

Curtis s'attendait à ce que son film fasse concurrence aux films d'Indiens produits par Hollywood en raison de l'authenticité des acteurs, des accessoires, des costumes, des danses et des activités. Cependant, le scénario, véhicule de cette authenticité, était confus; il présentait une histoire d'amour, d'intrigues et d'aventures compliquée. Dans War Canoes, les anthropologues ont remplacé les intertitres de Curtis par leurs propres intertitres elliptiques, ce qui rend l'histoire impossible à suivre. Le récit n'est plus qu'un complément bizarre des images qui ont très peu à voir avec les événements annoncés par les titres. Il en résulte un clivage radical entre le texte des acteurs et le texte de l'auteurréalisateur. Les anthropologues ont même ajouté une bande sonore en langue kwakiutle, sans offrir de traduction. Conséquemment, il semble que le film, appartenant aux Kwakiutls, soit entièrement différent de celui que perçoivent les spectateurs non kwakiutls. 
Le manque de continuité du récit et l'impression de doublage produite par la disjonction des images et des titres sont inhérents aux séquences originales de Headhunters. Le montage est minimal et les gros plans sont rarement utilisés dans ce film. Laction se déroule donc devant une seule caméra statique. Les compositions sont souvent mises en scène de façon frontale, gravitant autour d'un personnage central, sans exploitation du hors-champ. Bien que Holm et Quimby mentionnent avoir remonté une séquence avec un chevauchement temporel, il y a plusieurs autres exemples de mises en scène confuses indiquant que le film n'a pas été tourné dans un esprit de continuité et ne pouvait donc être "corrigé" sans un nouveau tournage.

D'autres conventions du cinéma des premiers temps sont encore apparentes dans War Canoes, notamment dans la scène de vision durant la première séquence du film, où Motana voit apparaître la figure de Naida dans la fumée du feu de camp alors qu'il rêve d'elle. De petits mouvements de caméra sont parfois utilisés pour recadrer l'action, bien que le cadre serve en général d'avantscène statique, n'offrant que peu de profondeur de champ. Le film restauré utilise les portraits que Curtis avait pris des acteurs principaux, dans leurs costumes, pour nous les présenter, ce qui n'est pas le cas du film original qui offre, lui, peu de gros plans permettant d'identifier les personnages. Toutes ces caractéristiques situent le film dans la période que Noël Burch qualifie de "primitive", avant le "déclin" du langage cinématographique qui a mené aux conventions limitées du réalisme narratif.

La théorie voulant que le cinéma des premiers temps soit "primitif" est un défi moderniste à l'hégémonie du mode de production institutionnalisé qui était plus ou moins "en place" en 1909 (Burch, p. 220). Durant les 10 ou 15 premières années du cinéma, la clôture narrative, le positionnement du spectateur et autres mécanismes de l'idéologie bourgeoise n'avaient pas encore été codifiés dans le langage du film. On a donc supposé que les réalisateurs et les spectateurs étaient plus «libres» d'utiliser le cinéma d'une façon qui, rétrospectivement, semble radicale. Cette interprétation du cinéma des premiers temps, influencée par le formalisme marxiste et l'esthétique avant-gardiste, présentait le cinéma d'avant Griffith comme un moment 


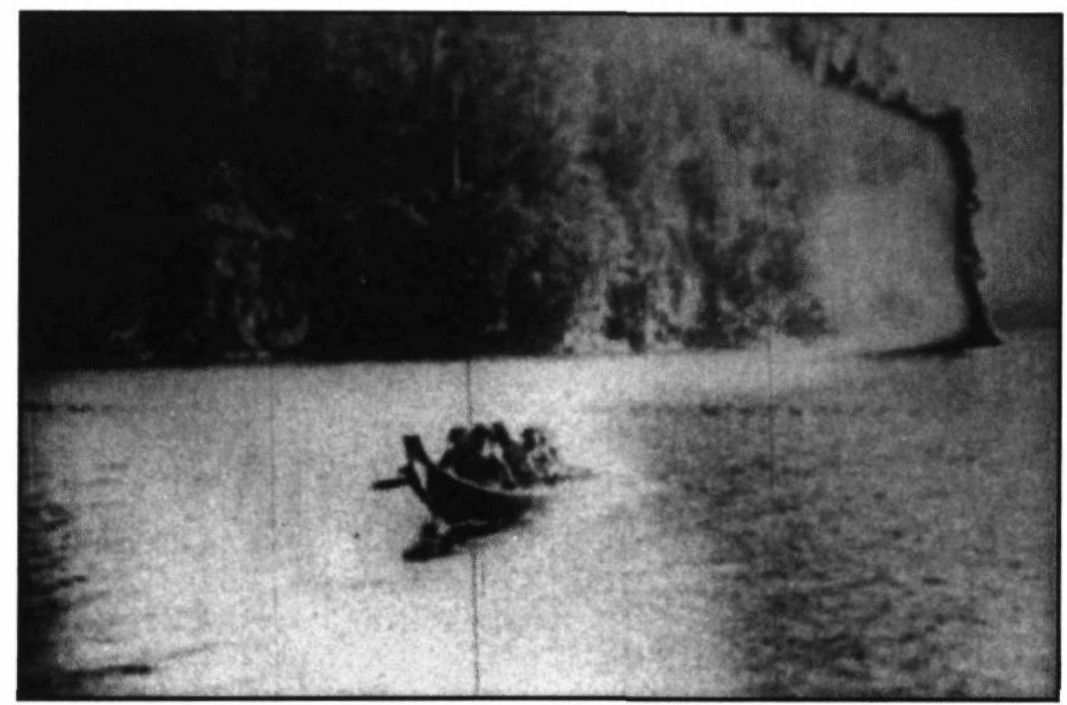

Land of the War Canoes

Solarisation de l'image dans la version restaurée du film, évocatrice de l'appropriation du cinéma des débuts par l'avant-garde.

préhistorique. Elle est comparable, à bien des égards, à l'élan qui porta l'ethnographie vers les cultures inchangées par l'histoire. Dans les deux cas, le discours primitiviste sert à idéaliser "l'autre " comme un être vierge que l'histoire a laissé intact.

Dans l'histoire du film ethnographique, il est bien possible que Headhunters soit le moment primitif, le style ethnographique primal, si politiquement incorrect qu'on peut facilement l'interpréter comme une allégorie des pratiques coloniales. La beauté du film réside dans son manque pervers d'authenticité, dans son approche théâtrale et ses artifices stylisés. Ce rare exemple d'ethnographie primitive nous donne l'occasion de replacer la poussée utopique du primitivisme dans un récit postcolonial. Si l'on arrivait à définir historiquement le point de convergence du cinéma primitif et du primitivisme ethnographique, l'historiographie utopique du discours primitiviste pourrait sans doute être rachetée allégoriquement, au-delà des préoccupations colonialistes et modernistes relatives à l'essence. Headhunters défie l'interprétation puriste du primitivisme, car il est indubitablement conçu pour offrir le primitif en spectacle. 
Son invention allégorique de l'altérité semble calquée sur rien de moins que le fantastique Voyage dans la lune (Méliès, 1902).

Il est cependant important de clarifier le contexte anthropologique de l'époque pour être en mesure d'apprécier le film dans toute sa "primitivité", car son apparence postmoderniste n'est que l'effet symptomatique d'une entreprise colonialiste fort contradictoire. L'exploitation du terme headhunting dans le titre et dans l'intrigue du film narratif de Curtis et la description détaillée des cérémonies qui interrompt le récit situent Headhunters dans la catégorie du "cinéma d'attractions" qui était presque disparu au moment de la réalisation du film. Tom Gunning a utilisé le terme "cinéma d'attractions", qui de toute évidence n'est pas dépourvu d'idéologie, en référence au cinéma des premiers temps. Parmi les actualités du cinéma des premiers temps, il existe tout un éventail de "film d'Indiens" portant des titres tels Teasing the Snakes (Edison, 1901) et Circle Dances (1898), dans lesquels des images exotiques d'Amérindiens sont exploitées pour le public blanc. C'est ainsi que les séquences des cérémonies navajoes et hopies, filmées par Curtis au début de sa carrière, ont abouti sur le marché commercial ${ }^{5}$.

Curtis voyait son travail comme une tâche urgente. Il s'était donné pour mission de documenter les cultures autochtones qui étaient, selon lui, au bord de l'extinction; ses prises de vue des autochtones sont toujours teintées d'un sens romantique du déclin. Dans Headhunters, l'absence de ce sens du déclin imminent devient alors significative. Curtis avait pourtant entrepris un projet imposant de récupération de la culture kwakiutle traditionnelle avant de commencer le tournage. L'architecture, les totems, les masques et les costumes: tout avait été préparé spécialement pour le film. Curtis avait soigneusement éliminé de la mise en scène tout signe de cultures externes afin de produire l'impression d'une culture autochtone vierge.

En tant que photographe, Curtis était célèbre pour son utilisation de costumes et d'accessoires servant à transformer en nobles sauvages des autochtones depuis longtemps dissociés de leur patrimoine ancestral (Lyman, 1982). Son ethnographie semble s'être développée en marge de l'anthropologie académique, comme un spectacle secondaire influencé par le marché du divertissement qui exploitait les curiosités aussi bien que par un engagement 


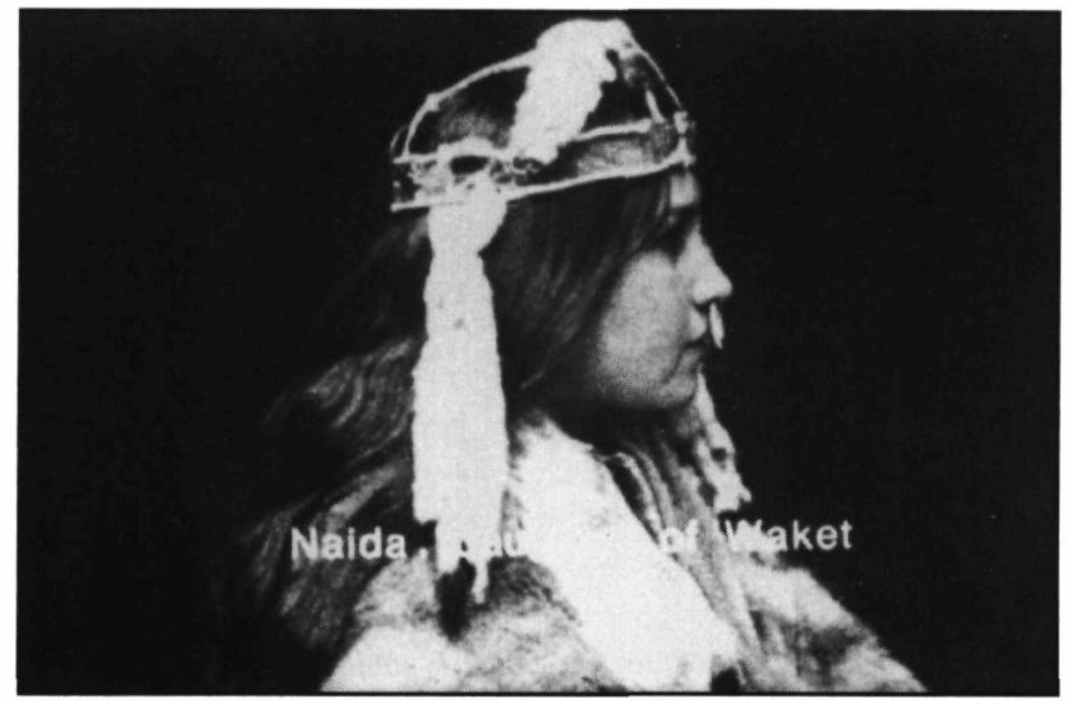

Land of the War Canoes

Un portrait typique de la prise de vues immobile de Curtis. Une série de portraits identifiant des personnages constitue une introduction

à la version restaurée du film.

sérieux vis-à-vis la documentation culturelle. Le sentiment d'urgence qui sous-tend le projet de Curtis provient d'une sensibilité romantique qui lui donnait droit à une certaine licence artistique pour mener à bien ce qu'il appelait son "art-science photographique». Toutefois, lorsque cette licence artistique s'est élargie au point d'incorporer un récit mélodramatique, l'image emblématique du déclin a cédé la place à un discours du désir.

La collection de têtes comme trophées de guerre était une tradition disparue de la culture kwakiutle depuis plusieurs générations; pourtant, les Indiens de Headhunters sont dépeints comme des sauvages à part entière. Ils brandissent triomphalement des têtes postiches et, dans quelques scènes, des crânes humains sont disposés de façon décorative dans le repère du méchant "sorcier". Vu la forte théâtralité du jeu et de la mise en scène, la chasse de têtes peut être interprétée comme un artifice narratif, une tradition que les descendants des guerriers kwakiutls ne pratiquent plus que de façon allégorique. Et c'est en regard à l'allégorie, dans la manière dont les Kwakiutls pratiquent leurs traditions, que le film résiste à l'autorité ethnogra- 
phique. Headhunters n'est pas un texte de deuil mais de triomphe du bien sur le mal, qui donne libre cours à la violence réprimée du noble sauvage de la photographie de Curtis.

La tentative de Curtis de dramatiser la culture kwakiutle représente en quelque sorte une reconnaissance de la subjectivité autochtone. Le mythe qu'il a conçu pour Headhunters est fortement imprégné de désirs, de répressions, de mises à l'épreuve et de conflits œdipiens ${ }^{6}$. Au lieu de présenter une énumération monotone de "données", ce qui a entrâné la mort du travelogue contemporain, Curtis a placé les autochtones au sein d'un discours du désir. L'histoire est de toute évidence calquée sur les scénarios contemporains de "ciné-romans", qui peuvent être considérés comme une importante représentation mythologique de la classe moyenne américaine. Mais elle permet néanmoins, en principe, au personnage autochtone Motana, d'assumer le rôle œdipien. Ainsi, du fait même que le langage de la représentation échappe à la codification du "mode de représentation institutionnalisé", l'espace subjectif n'est pas pleinement découpé.

Au lieu de cela, une subjectivité allégorique est conçue par l'entremise d'un style de jeu à plusieurs niveaux. Curtis dit avoir été attiré par les Indiens de la côte Ouest, car " [...] leurs cérémonies sont développées à un point tel que l'emploi du terme "dramatique" est pleinement justifié" (Curtis, cité dans Holm et Quimby, p. 31). En effet, les Kwakiutls possédaient tout un éventail de danses et de rôles que s'échangeaient les individus et les familles lors de cérémonies. Le système du potlatch régissait la distribution des richesses et cette structure économique était bien ancrée dans une structure dramatique composée de costumes, de masques, de danses, de rôles, et même de dialogues basés sur la mythologie. Le nom kwakiutl de la cérémonie d'hiver présentée vers la fin de Headhunters, "ts! e' ts! equa", signifient "être malhonnête, tricher" (Boas, p. 172).

Autrement dit, les Kwakiutls étaient des acteurs d'expérience. Curtis les a affublés de perruques et de costumes, leur permettant ainsi de personnifier leurs ancêtres plutôt qu'eux-mêmes. Toutefois, les acteurs n'ont pas pleinement rempli leurs rôles assignés, en partie parce que les personnages étaient trop étrangers à leur culture, mais aussi en raison du style "histrionique " 
de leur jeu. L'intégration au film de leurs propres danses cérémoniales pour lesquelles ils se costument en animal ou en oiseau avec masques, fourrures et plumes ne fait que souligner le sentiment de dédoublement du style de jeu.

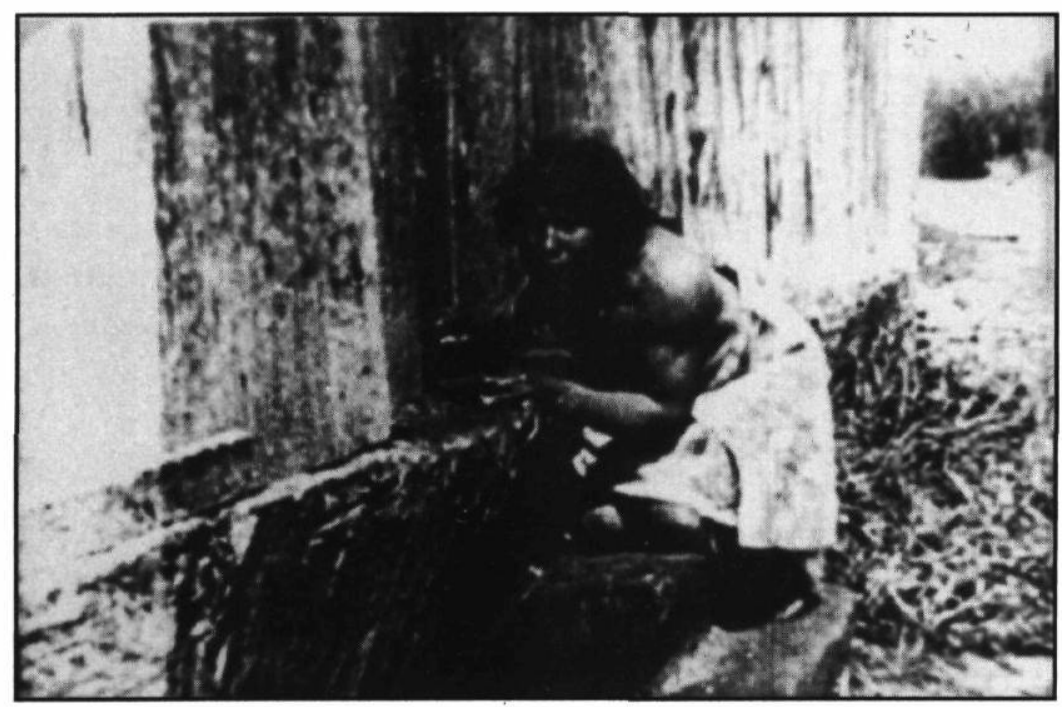

Land of the War Canoes

Motana sur le point de délivrer Naida de ses ravisseurs

Curtis affirme qu'il a dû négocier intensivement afin de distribuer les rôles, car il fallait déterminer si chaque acteur et actrice avait le "droit» de jouer le rôle qui lui était assigné ${ }^{7}$. En fin de compte, trois actrices ont interprété le principal personnage féminin, Naida, et un même acteur a joué deux des principaux rôles masculins. Dans les années 60 , lorsque Holm et Quimby ont présenté les séquences non montées de la version originale, certains spectateurs kwakiutls, reconnaissant les acteurs et actrices, ne pouvaient suivre l'intrigue qui identifiait arbitrairement les individus aux personnages (Holm et Quimby, p. 59). Dès lors que les Kwakiutls déchiffraient les images indexicalement plutôt que symboliquement, leur interprétation devenait résistante, contraire au grain métaphorique du texte.

Au lieu d'un récit cinématographique, il est possible que les Kwakiutls aient perçu un documentaire sur leur performance 
dans le film d'un Blanc. Le film constitue un rappel vivant des pratiques traditionnelles, parallèlement au confinement colonial inscrit dans les conventions rigoureuses du cadrage et du scénario. En tant que texte de mémoire culturelle, le film, dans sa forme, est allégorique, voire archéologique. Il expose plusieurs niveaux historiques de représentation. Le dialogue entre les acteurs de 1914 et la bande sonore de 1972 constitue en soi une correspondance historique vibrante; il témoigne de l'écart entre les générations, mais aussi du sentiment collectif qui les lie. En raison du mélange tout particulier de fiction et de documentaire caractérisant War Canoes, le film peut être lu et interprété de maintes façons. L'aspect fictionnel permet une interprétation différente ou "transposée" du documentaire, il prête au film une certaine ambiguïté et au spectateur, un contrôle accru.

C'est cette textualité ouverte de l'espace discursif de Headhunters qui distingue ce dernier du film auquel il est le plus souvent comparé, Nanook of the North (Flaherty, 1920). Ce n'est pas seulement le type d'histoire qui différencie Nanook de Headhunters mais, par-dessus tout, la forme narrative. Flaherty a choisi comme véhicule de son intimisme humaniste un récit rigoureusement monté, où les gros plans individualisent les autochtones en tant que personnages et où les plans d'ensemble situent ces personnages dans le décor pittoresque et radicalement exotique de l'Arctique. Une intégration soignée des titres et des images rend le récit naturel et prête un ton familier à la perspective colonialiste. Dans toute l'amplitude du "mode institutionnalisé" du système-narrateur, ou lecteur-film interne ${ }^{8}$, l'histoire de Nanook semble se raconter d'elle-même. La subjectivité inventée par Flaherty pour ses personnages est, toutefois, extrêmement limitée. Dans Nanook, le drame du combat féroce entre l'homme et la nature confere une certaine simplicité aux acteurs complètement identifiés aux rôles qu'ils interprètent.

Par son esthétisation stylistique de la vie autochtone, Nanook est en quelque sorte un équivalent cinématographique du romantisme photographique de Curtis. Le film se conforme parfaitement à la conjoncture moderniste où l'on reconnaît la pureté d'une culture et l'intégrité de l'autre ethnographique, tout en gardant cette culture à bonne distance. On rend cette 
distance sécuritaire en présentant la culture autochtone comme un élément échappant à l'histoire, en la situant éternellement dans le présent. Nanook et Headhunters brouillent tous deux la distinction entre les acteurs autochtones et leurs ancêtres. Cependant, l'échec de Headhunters en ce qui a trait au réalisme narratif - son primitivisme esthétique - incite à lire cette différence historique dans le cœur du film.

Force est de reconnaître l'habileté des spectateurs autochtones à dépasser, dans leur interprétation, le discours colonialiste inhérent aux films muets ethnographiques basés sur des paradigmes racistes. Les collectivités autochtones s'efforcent de préserver leur culture par la pratique d'activités et de rituels traditionnels, et ces films leur offrent une image sans pareil de la génération précédente poursuivant exactement le même but ${ }^{9}$. War Canoes représente un moment exceptionnel dans l'histoire du cinéma, car il est, en soi, si fortement intertextuel, tout en présentant le salvage paradigm comme une forme de désir narratif. Lidéal de l'autonomie et de l'intégrité culturelles est atteint dans le film de Flaherty comme dans celui de Curtis et, d'une certaine manière, ces films répondent tous deux au narcissisme cinématographique du spectateur: se voir soi-même, ou son groupe ethnique, occuper à l'écran un espace subjectif privilégié.

Lorsqu'on a demandé à Gloria Cranmer Webster, conservatrice kwakiutle du U'Mista Cultural Center, pourquoi les Kwakiutls avaient participé au film de Curtis avec tant d'enthousiasme, celle-ci a répondu, en toute simplicité, que c'était probablement très amusant. Si le film peut nous apprendre quelque chose sur l'ethnographie postmoderniste, c'est sans doute dans la réelle perversité de l'intertexte "fictionnel" de divertissement et du cinéma d'attractions "primitif" que résident ses enseignements. En tant que discours antiréaliste, il libère l'ethnographie du fardeau de l'autorité et du poids de l'historiographie moderniste du déclin. War Canoes est un film comportant plusieurs niveaux; il dépeint une collectivité autochtone bien en vie, attachée à son passé colonial aussi solidement qu'à son histoire précoloniale. Le film, dans son intertextualité et sa survie fragmentaire, représente aussi un exemple exceptionnel de document postmoderne de mémoire culturelle. 


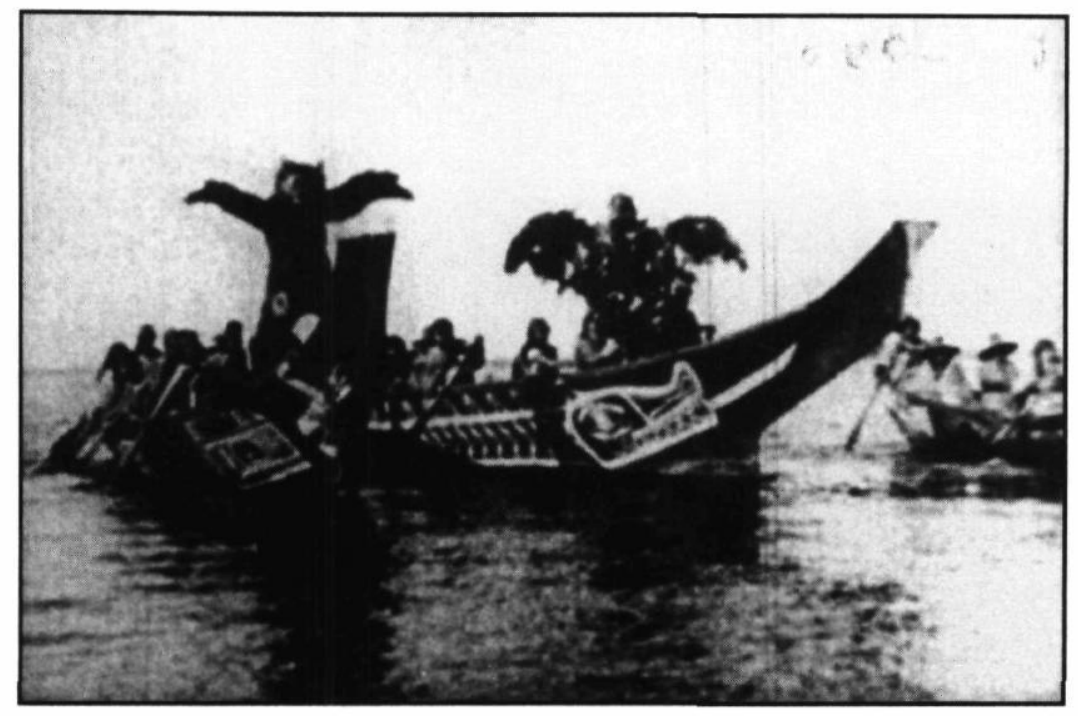

Land of the War Canoes

La procession cérémonielle des canots avec des hommes déguisés en oiseaux.

Les formes utopiques du désir, découlant de la convergence du "primitivisme" et du divertissement populaire, peuvent être associées à la mémoire culturelle. L'allégorie de la représentation souligne l'essence de ce qui ne peut être représenté, ce qui existe en marge du cadre de la caméra de l'homme blanc. Curtis devait composer avec un environnement chaotique dans lequel l'exploitation des cultures différentes rivalisait avec l'émergence des formes d'un réalisme narratif. Dans Headhunters, ce réalisme a été confondu avec l'objectivité scientifique, ce qui a produit une forme de divertissement essentiellement perverse qui ne peut être associée à l'esthétique réflexive du modernisme. En tant que film expérimental, Headhunters peut cependant servir de modèle pour une forme de représentation ethnographique postcoloniale et postmoderne.

Concordia University

Traduit de l'anglais par Josée Blanchet 


\section{NOTES}

1 Je désire remercier le Conseil de recherches en sciences humaines du Canada pour son appui, qui m’a permis de rédiger ce texte. Je remercie aussi Isabelle Raynauld, Diane Tegenkamp et Angela Fong. Une version plus longue de ce texte sera publiée en anglais dans la revue Visual Anthropology, vol. 8, $\mathrm{n}^{\circ} 1$.

2 L'expression salvage paradigm a été créée par James Clifford, pour exprimer l'allégorie ethnographique du sauvetage culturel, très répandue, qui exclut les autochtones de l'histoire moderne. Voir James Clifford et al., "Beyond the "Salvage " Paradigm", Discussion in Contemporary Culture, $\mathrm{n}^{\circ} 1$ (1987).

3 Headhunters a pris l'affiche à New York et à Seattle en 1914 et s'est maintenu à l'affiche dans différents cinémas à travers le pays jusqu'en 1915. Le film était coloré à la main et sa projection était accompagnée par un orchestre. Il a été financé par la société de production de Curtis, The Continental Film Company. L'investissement majeur était justifié par l'espoir que "[...] les films d'Indiens existeront jusqu’à la fin des temps en raison de leur importance historique et ethnologique. Ils ne tomberont pas dans l'oubli au bout de six mois; plutôt, leur valeur augmentera et le coût initial sera éventuellement recouvré" (Curtis, cité par Bill Holm et Paul Quimby, Edward S. Curtis in the Land of the War Canoes: A Pioneer Cinematographer in the Pacific Northwest, Vancouver: Douglas-McIntyre, 1980, p.113).

4 Cette information a été tirée de la collection des paperprints de la Library of Congress qui offre des descriptions de scénarios de films recueillis depuis 1914. À part les films de Griffith, il existe un bon nombre d'autres "films d'Indiens" dignes de mention: The Indian de Klaw et Elanger (1914), un film de 3 bobines qui met en vedette 250 figurants indiens, et The Tourists de Mack Sennett (1912), dans lequel Mabel Normand séduit sans le vouloir un chef indien.

5 The Shadow Catcher: Edward S. Curtis and the North American Indian, un film de T. C. McLuhan (1974), comporte des séquences de cérémonies hopies et navajoes tournées par Curtis.

6 Cette description de l'intrigue est basée sur le résumé que Holm et Quimby ont tiré de l'histoire originale (Edward S. Curtis in the Land of the War Canoes: A Pioneer Cinematographer in the Pacific Northwest, Vancouver: Douglas-McIntyre, 1980, p.42). Leur propres intertitres dans War Canoes ne reflètent que très partiellement le récit original.

7 Voir les propos de Curtis dans "Filming the Headhunters: How the Vanishing Race is Being Preserved in Moving Pictures", Strand Magazine (1915). Holm et Quimby commentent le manque de fiabilité de cet article, lequel en effet est rempli d'exagérations et de fausses représentations qui font de la réalisation du film une aventure anthropologique hautement exotique. Néanmoins, il est significatif que Curtis souligne les éléments dramatiques de la culture kwakiutle tels qu'il les percevait.

8 André Gaudreault cite un manuscrit inédit de Tom Gunning où il explique que : "Le système-narrateur semble " lire " les images à l'auditoire du simple fait qu'il les présente. Le narrateur est invisible et ne révèle sa présence qu’à travers sa manière de révéler les images à l'écran" ("Showing and Telling: Image and Word in Early Cinema ", dans Thomas Elsaesser (direction), Early Cinema: Space, Frame, Narrative, Londres: British Film Institute, 1990, p. 279, traduction de l'anglais). Gunning élaborera son analyse du système-narrateur dans un prochain ouvrage sur D. W. Griffith.

9 Le film de Claude Massot, Nanook Revisited (1990), comporte des scènes remarquables de spectateurs inuits visionnant Nanook avec enthousiasme, mais aussi avec un sens critique. 


\section{OUVRAGES CITÉS}

Boas, Frank. Kwakiutl Ethnography. Chicago: University of Chicago Press, 1966.

Burch, Noël. "A Primitive Mode of Representation? ", dans Thomas Elsaesser (direction), Early Cinema: Space, Frame, Narrative. Londres: British Film Institute, 1990, p. 220-227.

Clifford, James, Dominguez, Virginia et Minh-ha, Trinh T. "Beyond the "Salvage" Paradigm ". Discussions in Contemporary Culture, n" 1, (1987).

Clifford, James. "On Ethnographic Allegory", dans James Clifford et George E. Marcus (direction), Writing Culture: The Poetics and Politics of Ethnography. Berkeley: University of California Press, 1986, p. 98-121.

Gaudreault, André. "Showing and Telling: Image and Word in Early Cinema ", dans Thomas Elsaesser (direction), Early Cinema: Space, Frame, Narrative. Londres: British Film Institute, 1990, p. 274-281.

Holm, Bill et Quimby, George Irving. Edward S. Curtis in the Land of the War Canoes: A pioneer Cinematographer in the Pacific Northwest. Vancouver: Douglas \& McIntyre, 1980.

Lyman, Christopher M. The Vanishing Race and Other Illusions: Photographs of Indians by Edward S. Curtis. Washington D.C. : Smithsonian Institution Press, 1982.

Manganaro, Marc. "Textual Play, Power, and Cultural Critique: An Orientation to Modernist Anthropology", Modernist Anthropology: From Fieldwork to Text. Princeton: Princeton University Press, 1990, p. 3-50. 